\title{
Influência da posição do objeto na frequência de alcances manuais em lactentes com desenvolvimento típico
}

\author{
Influence of object position on the frequency of manual reaching \\ in typically developing infants
}

Jaqueline da Silva Frônio ${ }^{1}$,Luciana Matta de Andrade e Silva², Rodrigo Jardim Gonçalves ${ }^{2}$, Paula Silva de Carvalho Chagas ${ }^{3}$, Luiz Cláudio Ribeiro ${ }^{4}$

Estudo desenvolvido pelo NIEDI Núcleo Interdisciplinar de Estudos do Desenvolvimento Infantil na Faculdade de Fisioterapia da UFJF - Universidade Federal de Juiz de Fora - Juiz de Fora, MG, Brasil.

' Doutora em Ciências Médicas pela Universidade Estadual de Campinas (UNICAMP); Coordenadora do Núcleo Interdisciplinar de Estudos do Desenvolvimento Infantil (NIEDI) Professora Adjunta da Faculdade de Fisioterapia da Universidade Federal de Juiz de Fora (UFJF) - Juiz de Fora (MG), Brasil.

Fisioterapeutas graduados pela UFJF - Juiz de Fora (MG), Brasil.

${ }^{3}$ Doutora em Ciências da Reabilitação pela Universidade Federal de Minas Gerais (UFMG); Professora Adjunta da Faculdade de Fisioterapia da UFJF - Juiz de Fora (MG), Brasil.

${ }^{4}$ Doutor em Demografia pela UFMG; Professor Adjunto do Departamento de Estatística do Instituto de Ciências Exatas da UFJF - Juiz de Fora (MG), Brasil.

ENDERECO PARA CORRESPONDÊNCIA: Jaqueline da Silva Frônio - Universidade Federal de Juiz de Fora - Faculdade de Fisioterapia - Centro de Ciências da Saúde - Rua José Lourenço Kelmer, s/n - Campus Universitário - Bairro São Pedro - CEP 36036-900 - Juiz de Fora (MG), Brasil E-mail: jaqueline.fronio@ufjf.edu.br.

APRESENTAÇ̃̃O:

maio 2010

ACEITO PARAPUBLICAÇÃO

jan. 2011

FONTE DE FINANCIAMENTO: nenhuma.

\section{CONFLITO DE INTERESSES:} nada a declarar.

O presente estudo foi apresentado no XX Congresso Nacional da ABENEPI e no XVI Simpósio de Fisioterapia da UFSCar, em 2009. Estudo aprovado pelo Comitê de Ética e Pesquisa da Universidade Federal de Juiz de Fora, sob o parecer 093/2008.
RESUMO: O objetivo deste estudo foi verificar a frequência de alcances no período de três a cinco meses de idade e se há influência da posição de apresentação do objeto. Treze lactentes com desenvolvimento típico foram avaliados aos três, quatro e cinco meses, em supino, sendo o objeto apresentado na linha média e nas linhas axilares direita e esquerda. O procedimento foi filmado e analisado para registro do número de alcances realizados nas posições de apresentação. Para análise foram utilizados os testes de Friedman e Wilcoxon, considerando o nível de significância $\alpha=0,05$. As frequências médias de alcances aumentaram até o quinto mês, sendo encontrada diferença significativa entre o terceiro e o quinto $(p=0,011)$ e entre o quarto e o quinto meses $(\mathrm{p}=0,008)$. O número de alcances na linha média aumentou significativamente do terceiro para o quinto $(p=0,011)$ e do quarto para o quinto mês $(p=0,025)$, e houve tendência de diferenciação $(0,1>p>0,05)$ entre o terceiro e o quarto mês $(p=0,058)$. Aos três meses, encontrou-se tendência de diferenciação $(p=0,066)$ entre a frequência de alcances nas linhas axilar ( $D$ ou $E$ ) e média. Áos quatro e cinco meses, não foi encontrada diferença estatisticamente significativa entre as posições. Concluiu-se que a frequência de alcances aumenta significativamente de três a cinco meses e a posição de apresentação do objeto parece influenciá-la na fase inicial de aquisição desta habilidade.

Descritores: desenvolvimento infantil; lactente; desempenho psicomotor.

ABSTRACT: The aim of this study was to evaluate the frequency of reaching in the period of three to five months of age and if the object presentation position has an influence on it. Thirteen typically developing infants were assessed at three, four, and five months of age. The frequency of reaching was evaluated with the infants in the supine position, with the object presented at midline, right, and left axillary lines. The procedure was shot and further analyzed to determine all reaching numbers at the presentation positions. For analysis, the Friedman and Wilcoxon's tests were used, considering a 5\% significance level. The mean reaching frequencies increased at the fifth month, with a statistically significant difference being found between the third and fifth $(p=0.011)$ and between the fourth and fifth months $(p=0.008)$. The amount of reaching at midline also significantly increased between the third and the fifth $(p=0.011)$, the fourth and the fifth months $(p=0,025)$, and a trend towards differentiation was identified between the third and fourth months $(p=0,058)$. At three months, reaching frequency showed a trend towards differentiation $(p=0.066)$ between the axillary line (right or left) and at midline. No significant differences between the positions were identified at the fourth and fifth months. In conclusion, the frequency of reaching increases significantly from three to five months, and the object presentation position seems to influence the initial stage of acquisition of this skill.

KEYWoRDs: child development; infant; psychomotor performance. 


\section{INTRODUCÃO}

A compreensão do processo de aquisição e desenvolvimento das habilidades motoras no primeiro ano de vida tem sido alvo de muitos estudos na área de desenvolvimento motor. Entre estas habilidades, o alcance manual assume considerável importância, pois junto com a preensão e manipulação de objetos, permite a exploração do ambiente pelo lactente ${ }^{1,2}$.

Thelen et al. ${ }^{3}$ já observaram o comportamento de alcance em alguns lactentes típicos aos três aos quatro meses de idade, podendo este ser definido como o movimento direcionado do membro superior a um objeto uni ou bimanual, sendo finalizado quando a mão toca o objeto, não sendo necessária a sua preensão ${ }^{3}$.

Ao longo do desenvolvimento, características do ambiente e do organismo e exigências de tarefa são fatores que podem condicionar a aquisição e o controle de uma habilidade ${ }^{4,5}$. Segundo Newell et al. ${ }^{5}$, esses fatores podem ser considerados como restrições da ação e podem ser classificados como fatores de restrição intrínsecos e extrínsecos ao organismo $^{6,7}$.

Há na literatura estudos que investigaram a influência de algumas restrições extrínsecas no desenvolvimento da habilidade de alcançar objetos. Rocha et al. ${ }^{8}$ verificaram que as propriedades físicas do objeto influenciam os ajustes necessários para realizar o alcance, e que objetos maleáveis favorecem a realização de alcances seguidos de preensão quando comparados a objetos rígidos. Coelho ${ }^{9}$ sugeriu em seu estudo que alvos com contraste de cor e estáticos promovem maior número de alcances, do que os sem contraste e em movimento. Fatores, como a posição corporal durante o movimento de alcance, também foram investigados em outros estudos. Carvalho et al. ${ }^{10}$ verificaram que a postura sentada propicia maior frequência de alcances em lactentes de quatro a seis meses quando comparada com a postura supina. Fallang et al. ${ }^{11}$ avaliaram a relação entre controle postural e alcance na postura supina em lactentes de quatro e seis meses de idade e sugeriram que uma melhora no desempenho do alcance está relacionada, em parte, à melhora no controle postural. Distância, localização e orientação do objeto também são fatores considerados de restrição extrínseca e podem influenciar a trajetória do braço em direção ao objeto ${ }^{12-14}$, assim como a frequência de alcances.

Estudos recentes ${ }^{15-17}$ encontrados na literatura, que apresentaram o objeto em diferentes posições, tiveram como objetivo avaliar a preferência manual e não a relação desta posição com a frequência de alcance. Assim, não foram encontrados estudos que tiveram como objetivo investigar a influência da posição do objeto na frequência de alcances em lactentes.

Com base nestas informações, o estudo teve como objetivo verificar a frequência de alcances manuais e se houve influência da posição de apresentação do objeto nesta frequência durante o período de aquisição desta habilidade em lactentes com desenvolvimento típico.

Ao ser investigada a frequência de alcances manuais em lactentes com desenvolvimento típico aos três, quatro e cinco meses de vida, apresentando o objeto em diferentes posições (linhas média e axilares), as seguintes hipóteses foram estabelecidas: no início da aquisição desta habilidade, a apresentação nas linhas axilares facilitará a ocorrência desse movimento por exigir menos ajustes e coordenação e por sofrer menor influência da ação da força de gravidade do que quando este for apresentado na linha média. Com o passar dos meses, ao praticarem essa atividade, os lactentes aprimorarão o alcance, o que permitirá a realização da maior frequência de alcances, independente da posição de apresentação do objeto.

\section{METODOLOGIA}

Foi realizado um estudo longitudinal, prospectivo, de lactentes com desenvolvimento típico, sem intercorrências, que pudessem levar a alterações no desenvolvimento, avaliados aos três, quatro e cinco meses de idade, período em que normalmente compreende a aquisição do alcance e a incorporação dessa habilidade em lactentes típicos. Os participantes e o tamanho da amostra foram escolhidos por conveniência, advindos das Unidades de Saúde do município de Juiz de Fora, em Minas Gerais. Na literatura consultada de estudos semelhantes, não foi encontrado cálculo amostral, mas o número de participantes variou entre 4 e 12 lactentes s, $^{8,11}$.

Os lactentes foram selecionados por meio da análise dos prontuários de três Unidades Básicas de Saúde (UBS) do município. Foram convidados a participar deste estudo, por intermédio de seus responsáveis, 43 lactentes que estavam com três meses no período programado para o recrutamento dos participantes (que durou três meses), dos quais apenas 16 compareceram à avaliação inicial (aos três meses). Destes, dois foram excluídos por serem prematuros (idade gestacional $<37$ semanas) e um por chorar durante o procedimento, de modo a não permitir a realização do teste. Sendo assim, 13 lactentes participaram da primeira avaliação aos três meses de idade, oito do sexo feminino e cinco do masculino, nascidos a termo $(39,08 \pm 1,25)$ e com índice de Apgar igual ou superior a 8 no primeiro e quinto minutos. Desses, quatro não compareceram às avaliações subsequentes, portanto, nove lactentes participaram das avaliações aos quatro e cinco meses.

Para a comparação mês a mês da frequência de alcances, segundo a posição de apresentação do objeto (linhas média e axilar), foram considerados os dados de todos os participantes que compareceram em cada mês estudado, isto é, 13 lactentes aos três meses e 9 lactentes aos quatro e cinco meses. Para a análise das mudanças ao longo do tempo (frequência de alcances totais e na linha média), foram considerados apenas os dados dos nove lactentes que compareceram às três avaliações programadas.

O estudo foi aprovado pelo Comitê de Ética e Pesquisa da Instituição, parecer 093/2008, e os pais ou responsáveis pelos lactentes assinaram previamente o termo de consentimento livre e esclarecido.

As avaliações foram realizadas em uma sala de consultas de cada uma das UBS participantes, com boa iluminação e com o mínimo de interferências visuais e/ ou auditivas possíveis. Os lactentes foram posicionados na maca pelas mães em decúbito supino, e estas permaneciam na sala em um local onde os lactentes 
não pudessem vê-las, porém podiam acompanhar o procedimento. Era dado 10 segundos para que os lactentes se adaptassem quando colocados nessa postura, sendo que dois pesquisadores permaneciam próximos a eles durante todo o procedimento para que não houvesse risco de quedas. Investigou-se a frequência de alcance em supino, por ser esta a postura que lactentes de culturas ocidentais permanecem, nesta faixa etária, a maior parte do tempo em casa e, por isso, é nela que normalmente adquirem experiência nessa habilidade ${ }^{11}$.

Para a realização deste estudo, foram utilizados um objeto maleável pequeno e colorido ("pompom" de lã antialérgica, $5 \mathrm{~cm}$ de diâmetro ${ }^{8}$, pendurado em um pequeno barbante), uma câmera filmadora digital (JVC ${ }^{\circledR}$ - DV Camcorder GY-DV 300), um tripé, uma maca e um cronômetro. O pompom foi escolhido como objeto, devido a pesquisas anteriores que verificaram que objetos maleáveis e coloridos facilitam o alcance ${ }^{8,9}$.

O objeto foi apresentado por um dos pesquisadores, por dois minutos, em cada uma das três diferentes posições (linhas axilar direita, axilar esquerda e média), na altura do seu manúbrio (linha horizontal), a uma distância correspondente ao comprimento do membro superior do lactente (distância entre o ombro e o punho, que foi mensurada antes do início do teste) (Figura 1). Considerou-se a linha média como uma linha vertical, a qual divide o corpo em duas metades equidistantes e as linhas axilares anteriores como sendo linhas verticais que separam o tronco e o membro superior. O objeto, suspenso pelo barbante, era apresentado e, caso o lactente não demonstrasse interesse, o mesmo era agitado no seu campo visual e movido até a posição programada (linhas axilar direita, axilar esquerda ou média). Quando o lactente fixava o olhar e direcionava a mão para o objeto, estando na posição programada, este permanecia imóvel. Caso o lactente apreendesse o objeto ou ficasse em contato com ele por mais de cinco segundos, este era retirado de sua mão e novamente apresentado na mesma posição até que se atingisse o tempo de dois minutos, previsto para aquela posição de apresentação. Entre uma posição e outra, era dado um intervalo de cinco segundos. Sendo assim, o tempo total do procedimento era de 6 minutos e 20 segundos. Este procedimento foi definido com base em estudos anteriores que investigaram o alcance em lactentes ${ }^{8,10,18}$. Para que a ordem de posicionamento do objeto não influenciasse nos resultados, este foi apresentado em diferentes sequências aleatoriamente pré-determinadas para cada lactente. $\mathrm{O}$ procedimento era aplicado apenas uma vez em cada lactente, em cada idade (três, quatro e cinco meses).

O procedimento foi filmado e cronometrado por outro pesquisador. A câmera filmadora foi posicionada posterossuperiormente ao lactente e fixada em um tripé, de forma a permitir a melhor visualização simultânea dos membros superiores e do objeto.

As avaliações coincidiram com a data de aniversário dos lactentes, aceitando uma variação de sete dias antes ou após essa data. O teste foi realizado preferencialmente entre as mamadas, e o lactente deveria estar em estado de alerta ativo.

A frequência de alcances foi calculada para cada posição de apresentação do objeto por meio da análise dos vídeos. Para isso, considerou-se como alcance o movimento realizado com uma ou duas mãos (uni ou bimanual), quando o olhar do lactente estava simultaneamente voltado para o objeto, resultando no contato da mão com o mesmo. Dois pesquisadores fizeram esta análise de forma independente. Para maior confiabilidade dos dados, foi realizado um estudo piloto e o treinamento dos pesquisadores, tendo sido obtido o índice de concordância intraclasse (ICC) de $0,93^{19}$. Os dados coletados foram registrados em um cartão individual e, posteriormente, em planilhas de arquivos do programa SPSS 14.0. Como os dados não satisfaziam aos critérios de normalidade, segundo o teste Kolmogorov-Smirnov, para a análise estatística foram utilizados os testes não-paramétricos de Friedman e Wilcoxon, considerando um nível de significância $\alpha=0,05$.

O teste de Friedman foi aplicado para comparar os postos médios das frequências de alcances totais (soma das frequências nas três posições) e na linha média ao longo dos três meses estudados (três, quatro e cinco meses). Como já citado, nesta análise, foram considerados apenas os dados dos nove lactentes que compareceram às três avaliações programadas. Para detectar entre quais meses ocorreram as diferenças encontradas pelo teste de Friedman, foi usado o teste de Wilcoxon.

Para a comparação dos postos médios das frequências de alcances em cada posição de apresentação do objeto (linhas axilar e média), aplicou-se o teste de Wilcoxon. Para esta análise, foram considerados os dados dos participantes que compareceram em cada mês estudado, isto é, 13 lactentes aos três e nove lactentes aos quatro e cinco meses. Estudos anteriores ${ }^{15,16,20}$ sugerem que já existe uma possível preferência manual nesta faixa etária, o que indicaria preferência por uma das linhas axilares. Como a verificação disto não foi objeto de investigação na presente pesquisa, para análise da influência da posição do objeto, optou-se por considerar apenas a linha axilar (D ou E) de maior frequência de alcances de cada participante em cada mês, anulando assim a possível interferência de uma preferência precoce.

\section{RESULTADOS}

A frequência média (desvios padrão) de alcances dos participantes do estudo aos três, quatro e cinco meses foi, respectivamente, 16,33 (10,12), 31,44 (23,33) e $58,00(24,28)$. A Figura 2 ilustra o total de alcances dos participantes, sendo observado, por meio do teste de Friedman, um

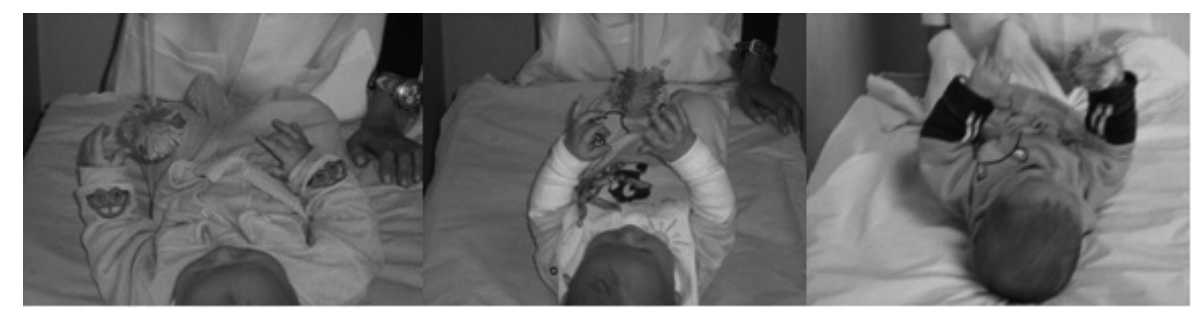

Figura 1. Posições de apresentação do objeto (linhas axilar esquerda, média e axilar direita) 
aumento significativo com o passar dos meses $(p=0,003)$. O teste de Wilcoxon indicou que estas diferenças ocorreram entre o terceiro e quinto mês $(p=0,011)$ e entre o quarto e quinto mês ( $p=0,008)$.

A Figura 3 ilustra a frequência de alcances realizados na linha média pelos participantes. Os valores das médias (desvio padrão) aos três, quatro e cinco meses foram respectivamente $4,56(4,66), 12,33(7,89)$

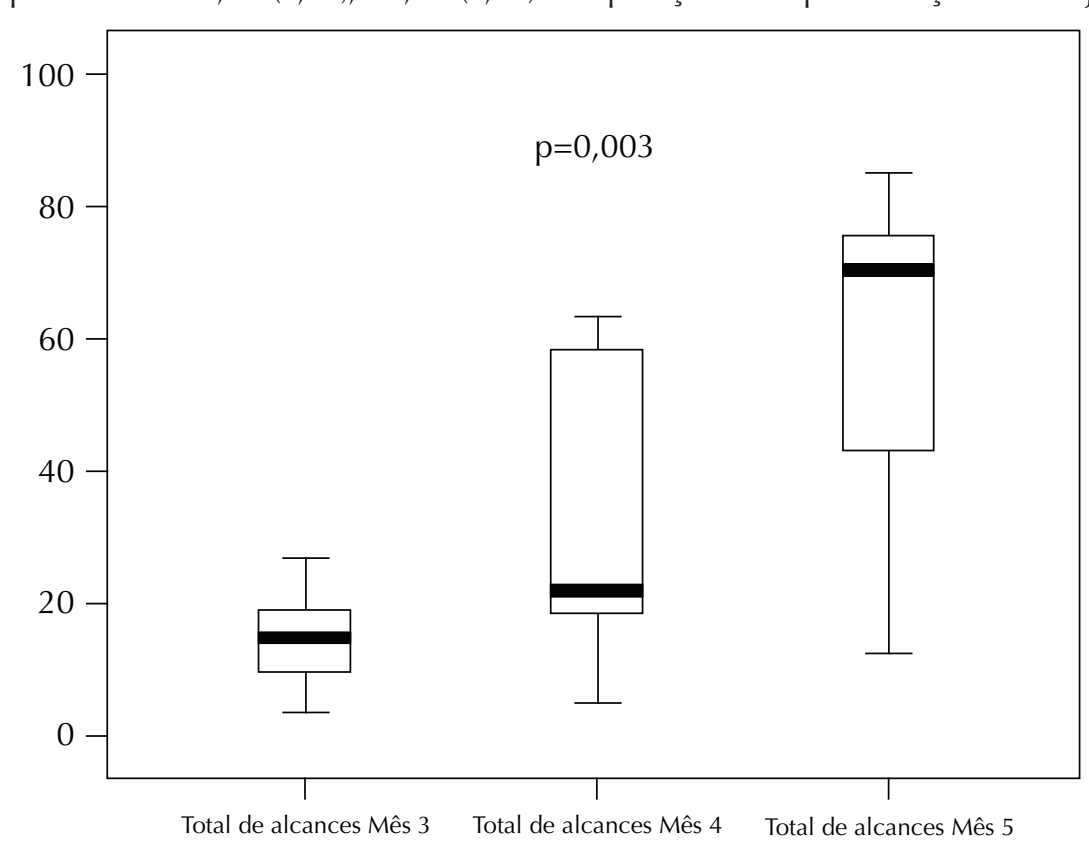

Figura 2. Box-plot da distribuição da frequência total de alcances e valor $p$ (Friedman) aos três, quatro e cinco meses (com base nos dados dos nove participantes que compareceram nos três meses estudados)

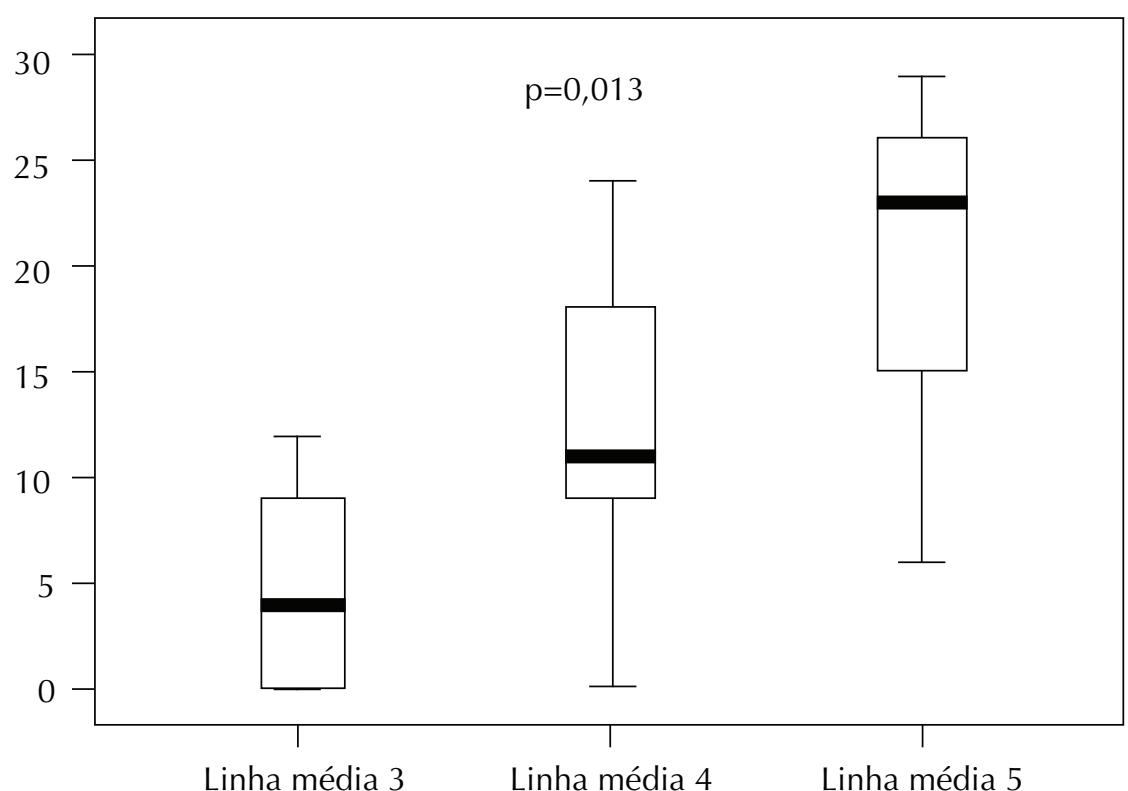

Figura 3. Box-plot da distribuição da frequência de alcances na linha média e valor p (Friedman) aos três, quatro e cinco meses (com base nos dados dos nove participantes que compareceram nos três meses estudados) (linhas axilar e média), encontra-se representada na Figura 4. Aos três meses, o teste de Wilcoxon indicou uma tendência de diferenciação $(p=0,066)$ entre as linhas axilar e média. Um dado a ser destacado é que, dos 13 lactentes avaliados, 5 não realizaram alcance na linha média nesta idade, mas todos realizaram alcances nas linhas axilares D ou E. Já aos quatro meses, não foi encontrada diferença significativa na frequência de alcances entre as linhas axilar e média, segundo o teste de Wilcoxon $(p=0,953)$. Dos nove lactentes avaliados, apenas um não realizou alcance na linha média e todos realizaram nas linhas axilares D ou E. Aos cinco meses, o teste de Wilcoxon também não revelou diferença estatisticamente significativa $(p=0,944)$ na frequência de alcances entre as posições de apresentação do objeto.

\section{DISCUSSÃO}

Este estudo acrescenta importantes considerações em relação ao período inicial de aquisição do alcance e aos aspectos que podem interferir neste processo. Não foram encontrados estudos que tiveram como objetivo investigar a influência da posição do objeto, na frequência de alcances em lactentes nesta ou em qualquer outra faixa etária. Os estudos encontrados, nos quais o objeto foi apresentado em diferentes posições (linha média e laterais), objetivavam analisar a preferência manual, a realização de alcances uni ou bimanuais e os que cruzavam a linha média ${ }^{15,16,20,21}$. Já os estudos que investigaram frequência de alcances não utilizaram diferentes posições de apresentação do objeto (somente linha média), investigando outros fatores influenciadores dessa habilidade (posição corporal, estabilidade postural, tamanho, rigidez e movimentação do objeto, dentre outros) $)^{8,11,22-26}$. Desta forma, os dados aqui encontrados não puderam ser diretamente comparados com os de outros estudos.

No presente estudo, aos três meses de idade foi encontrada uma média de aproximadamente 16 alcances por lactente, número que quase dobrou aos quatro meses, sendo que todos realizaram alcances nos meses estudados. Estes dados não corroboram a literatura, na qual a maioria dos estudos investiga o alcance em idades posteriores por considerar que esse comportamento se inicia só aos 
quatro meses e que, mesmo nessa idade, uma quantidade significativa de lactentes ainda não o realiza $3,6,11,27,28$. Um dos aspectos que pode ter contribuído para esta diferença é o fato do objeto ter sido apresentado nas linhas axilares (D e E).

Apesar da grande diferença entre as médias de alcances entre o terceiro e o quarto mês, isto não representou significância estatística, o que pode estar relacionado à grande variabilidade encontrada entre os participantes na fase de aquisição da habilidade, verificada pelos altos valores do desvio padrão. Esta variabilidade também pode ter influenciado os achados com relação aos alcances na linha média nestes meses.

Apesar de todos os lactentes (13) avaliados aos três meses terem realizado o alcance nas linhas axilares (D ou E), cinco não o fizeram na linha média. Assim, ainda que a análise estatística tenha encontrado apenas tendência de diferenciação entre as linhas axilar e média, é possível perceber que, para os lactentes que ainda não eram capazes de realizar o alcance na linha média, a apresentação do objeto na linha axilar facilitou o alcance no início da sua aquisição. Isto reforça a primeira hipótese do presente estudo, que sugere que a apresentação nas linhas axilares facilita este movimento por exigir menos ajustes, menos coordenação e por sofrer menor influência da ação da gravidade do que na linha média.

Aos quatro meses, dos nove lactentes avaliados, apenas um não realizou o alcance na linha média e todos realizaram nas linhas axilares (D ou E). Como já abordado, este dado difere dos estudos citados $^{3,6,11,27,28}$, que relataram haver um número significativo de lactentes nessa faixa etária que ainda não realizaram alcances na linha média. Este fato pode estar relacionado ao objeto usado no estudo (maleável e colorido), o qual, segundo estudos anteriores ${ }^{8,9}$, facilita o movimento de alcance ou ainda ser devido ao fato de que, como essas crianças foram avaliadas primeiramente aos três meses, na presença das mães/responsáveis, pode ter ocorrido um incentivo adicional à prática espontânea em suas casas, muitas vezes encorajada pelos pais. Segundo Gibson e Pick ${ }^{29}$, os pais mudam o ambiente social quando percebem que os lactentes estão respondendo aos seus estímulos. Assim, se os lactentes demonstram interesse ou intenção de alcançar um brinquedo, os pais tendem a mostrá-lo mais frequentemente.

Aos cinco meses de idade, todos os lactentes avaliados realizaram alcances nas três posições de apresentação, não havendo diferença estatisticamente significativa de frequência entre elas. Este achado confirma a segunda hipótese do estudo, a qual supõe que, com o avançar da idade e o treino da habilidade, a frequência de alcances aumenta independente da posição de apresentação do objeto. Esta hipótese também é reforçada pelo aumento progressivo e significativo, que ocorreu dos três aos cinco meses na frequência total de alcances e na linha média. Este achado está de acordo com diversos autores $3,8,22,30$, os quais sugerem que os lactentes melhoram suas habilidades de alcançar e realizam um maior número de alcances com o avançar da idade.

O presente estudo possui algumas limitações como o diferente número de participantes em cada mês (devido a desistências) e o fato de não terem sido investigadas outras posições corporais (sentado e reclinado). Apesar disso, oferece informações importantes para o possível embasamento de técnicas de intervenção precoce e de orientações para os pais, e para o aprofundamento de investigações acerca do período inicial de aquisição dessa habilidade.

\section{CONCLUSÃO}

Os resultados indicaram que a habilidade de alcance já está presente aos três meses em lactentes típicos e que a posição de apresentação do objeto parece influenciar sua frequência na postura supina, facilitando a ocorrência do mesmo quando o objeto é apresentado nas linhas axilares em sua fase inicial de aquisição. Indicam ainda que há um aumento progressivo no número de alcances com o passar dos meses, independente da posição de apresentação do objeto.

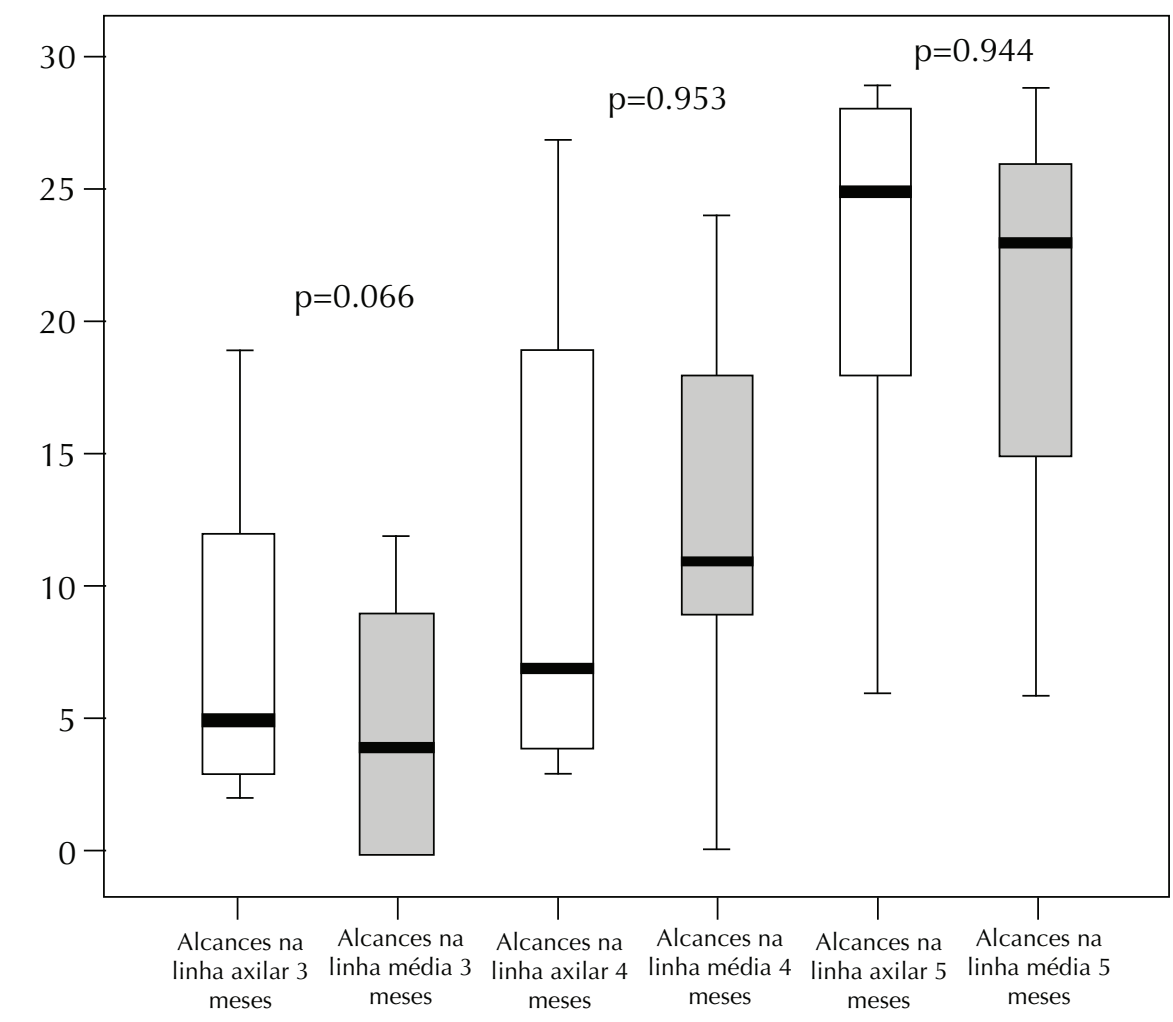

Figura 4. Box-plot da distribuição da frequência de alcances nas linhas axilar e média e valor $\mathrm{p}$ (Wilcoxon) aos três, quatro e cinco meses de idade (com base nos dados dos 13 participantes que compareceram aos três meses e dos 9 que compareceram aos quatro e cinco meses) 


\section{REFERÊNCIAS}

1. Thelen E, Corbetta D, Kamm K, Spencer JP, Schneider $\mathrm{K}$, Zernicke RF. The transition to reaching: mapping intention and intrinsic dynamics. Child Dev. 1993;64(4):1058-98.

2. Fallang B, Saugstad OD, Hadders-Algra M. Postural adjustments in preterm infants at 4 and 6 months postterm during voluntary reaching in supine position. Ped Res. 2003;54:826-33.

3. Thelen E, Corbetta D, Spencer JP. Development of reaching during the first year: role of movement speed. J Exp Psychol Hum Percept Perform. 1996;22(5):1059-76.

4. Lopes VB, Lima CD, Tudella E. Motor Acquisition Rate in Brazilian Infants. Inf Child Dev. 2009;18:122-32.

5. Newell KM, Liu YT, Mayer-Kress G. A dynamical systems interpretation of epigenetic landscapes for infant motor development. Infant Behav Dev. 2003;26:449-72.

6. Carvalho RP, Tudella E, Caljouw SR, Savelsbergh GJ. Early control of reaching: effects of experience and body orientation. Infant Behav Dev. 2008;31(1):23-33.

7. Campos AC, Rocha NACF, Savelsbergh GJ. Development of reaching and grasping skills in infants with Down syndrome. Res Dev Dis. 2010;31:70-80.

8. Rocha NA, Silva FP, Tudella E. The impact of object size and rigidity on infant reaching. Infant Behav Dev. 2006;29(2):251-61.

9. Coelho ZAC. O impacto da informação ambiental no desenvolvimento do alcance em crianças nascidas a termo, na faixa etária de 4 a 5 meses: uma abordagem ecológica. Escola de Educação Física, Fisioterapia e Terapia Ocupacional UFMG; 2004.

10. Carvalho RP, Tudella E, Savelsbergh GJ. Spatiotemporal parameters in infant's reaching movements are influenced by body orientation. Infant Behav Dev. 2007;30(1):26-35.

11. Fallang B, Saugstad OD, Hadders-Algra M. Goal directed reaching and postural control in supine position in healthy infants. Behav Brain Res. 2000;115(1):9-18.

12. Bhat AN, Heatheotk JC. Calkiwav JC. Toy-oriented changes In hand and joint kinematics during the emergence of purposeful reaching. Infant Behav Dev. 2005;28:445-65.

13. Bhat N. Galloway JC. Toy-oriented changes during early arm movements: hand kinematics. Infant Behav Dev. 2006;29:358-72.

14. Lee, MH, Liu YT, Newell KM. Longitudinal expressions of infant's prehension as a function of object properties. Infant Behav Dev. 2006;29:481-93.

15. Morange F, Peze A, Bloch H. Organization of left and right hand movement in a prehension task: $A$ longitudinal study from 20 to 32 weeks. Laterality. 2000;5(4):351-62.
16. Morange F, Bloch $\mathrm{H}$. Lateralization of the approach movement end prehension movement in infants from 4 to 7 months. Early Develop Par. 1996;5(2):81-92.

17. Fagard J, Spelke E, von Hofsten C. Reaching and grasping a moving object in 6-, 8-, and 10-month-old infants: Laterality and performance. Infant Behav Dev. 2009;32:137-46.

18. Corbetta D, Thelen E, Johnson K. Motor constraints on the development of perception-action matching in infant reaching. Infant Behav Dev. 2000;23:351-74.

19. Almeida KM, Dutra MVP, Mello RR, Reis ABR, Martins OS. Validade concorrente e confiabilidade da Alberta Infant Motor Scale em lactentes nascidos prematuros. J Pediatr (Rio J). 2008;84(5):442-8.

20. Corbetta D, Williams J, Snapp-Childs W. Plasticity in the development of handedness: evidence from normal development and early asymmetric brain injury. Dev Psychobiol. 2006;48(6):460-71.

21. Van Hof P, van der Kamp, Savelsbergh GJ. The Relation of Unimanual and Bimanual Reaching to Crossing the Midline. Child Dev. 2002;73(5):1353-62.

22. Bhat AN, Lee HM, Galloway JC. Toy-oriented changes in early arm movements, II: joint kinematics, Infant Behav Dev. 2007;30:307-24.

23. Fagard J. Linked proximal and distal changes in the reaching behavior of 5- to 12-month-old human infants grasping objects of different sizes. Infant Behav Dev. 2000;23:317-29.

24. Van Hof P, van der Kamp J, Caljouw S, Savelsbergh GJ. The confluence of intrinsic and extrinsic constraints in 3- to 9-month-old infant's catching behavior. Infant Behav Dev. 2005;28:179-93.

25. Rocha NACF, Costa CSN, Savelsbergh G, Tudella E. The effect of additional weight load on infant reaching. Infant Behav Dev. 2009;32:234-7.

26. Van Hof P, van der KJ, Savelsbergh GJ. Three- to eightmonth-old infants' catching under monocular and binocular vision. Hum Mov Sci. 2006;25(1):18-36.

27. Van der Fits IBM, Klip AWJ, van Eykern LA, HaddersAlgra M. Postural adjustments during spontaneous and goal-directed arms movements in the first half year of life. Behav Brain Res. 1999;106:75-90.

28. Carvalho RP. A influência da postura corporal no movimento de alcance manual em lactentes de 4 meses de vida. Universidade Federal de São Carlos; 2004.

29. Gibson EJ, Pick AD. An ecological approach to perceptual learning and development. New York: Oxford University Press; 2000. p. 15-25.

30. Bakker H, Graaf-Peters VB, van Eykern LA, Otten B, Hadders-Algra M. Development of proximal arm muscle control during reaching in young infants: From variation to selection. Infant Behav Dev. 2010;33:30-8. 